\title{
Fractional Order Generalized Thermoelastic Problem in a Thick Circular Plate with Periodically Varying Heat Source
}

\author{
Authors: J.J.Tripathi ${ }^{1}$, K.C. Deshmukh², J. Verma ${ }^{3}$ \\ ${ }^{1}$ Department of Mathematics, Dr. Ambedkar College, Deekshabhoomi, Nagpur-440010, Maharashtra, India. \\ ${ }^{2}$ Department of Mathematics, RashtrasantTukdojiMaharaj Nagpur University, Nagpur-440033, Maharashtra, India. \\ ${ }^{3}$ Department of Mathematics,Pillai HOC College of Engineering \& Technology, Rasayani, Navi Mumbai, India
}

Received 31 May 2016, Revised 26 June 2017, Accepted 26 June 2017

\begin{abstract}
This paper is concerned with fractional order thermoelastic response due to a heat source whose magnitude varies periodically with time within the context of generalized thermoelasticity with one relaxation time. Traction free boundary conditions are considered and the thick circular plate is subjected to a given axisymmetric temperature distribution. Integral transform technique is used to derive the solution in the transformed domain. Laplace transforms are inverted using a numerical scheme. Mathematical model is prepared for Copper material and results for temperature, displacement and stress distributions are computed and represented graphically.
\end{abstract}

Keywords: Fractional order; thermoelastic; thick plate; relaxation time; heat source.

\section{Introduction}

In 1967, Lord and Shulman [1] were the first to generalize Biot's theory of coupled thermoelasticity. This theory ensures finite speed of propagation for waves. Due to the experimental validation available in favor of the finite speed of propagation of heat waves, generalized thermoelasticity theory is receiving serious attention.

Mallik and Kanoria [2] studied the two dimensional problem in generalized thermoelasticity for a transversely isotropic thick plate having heat source. A two dimensional problem for a half space and thick circular plate with heat sources have been solved by El-Maghraby [3]. Tripathi et al. [4] discussed the temperature distribution and thermal stresses in a semi-infinite cylinder whose upper and lower surfaces are traction free and subjected to a given axisymmetric temperature distribution within the context of Lord-Shulman theory. Recently, Tripathi et al. [5, 6] studied problems for a thick circular plate and a half space within the context of generalized thermoelastic diffusion with one relaxation time.

During recent years, several interesting models have been developed by using fractional calculus to study the physical processes particularly in the area of heat conduction, diffusion, mechanics of solids, electricity, etc. Various types of definition and approaches of fractional order derivatives have become the main aim of many researchers. The reason behind introduction of the fractional theory is that it predicts retarded response to physical stimuli, as is found in nature, as opposed to instantaneous response predicted by the generalized theory of thermoelasticity. Povstenko [7-10] studied various applications of theory of the fractional order thermoelasticity. Youssef [11] established the fractional order generalized thermoelasticiy in the context of thermoelasticity with one relaxation time. Ezzat and Karamany $[12,13]$ established a new model of fractional heat conduction equation based on Taylor's series expansion of time fractional order. Sherief et al. [14] also established a new model by using the Lord-Shulman model of generalized thermoelasticity. Bachher [15] discussed the deformation due to periodically varying heat sources in a reference temperature dependent thermoelastic porous materials with a time fractional heat conduction law. Islam and Kanoria [16] dealt with the problem of magnetothermoelastic interaction in a perfectly conducting elastic medium where the boundary is stress free and subjected to a thermal loading in the context of fractional order two temperature generalized thermoelasticity. Raslan [17] studied the fractional order theory of thermoelasticity to the two dimensional problem of thick plate whose lower and upper surfaces are traction free and subjected to a given axisymmetric temperature distribution in the context of Lord-Shulman theory. Tripathi et al. [18], dealt with a generalized thermoelasticproblem of mass diffusion in a thick circular plate under the effect of a heat source. Sarkar [19], studied the wave propagation in an initially stressed elasticsolid half-space under time-fractional order twotemperature magneto-thermoelasticity. Tripathi et al. [20] discussed a dynamic problem of fractional order thermoelasticity for a thick circular plate with finite wave speeds. Warbhe et al. [21], studied fractional order heat conduction and its associated thermal stresses in a thin circular plate with constant temperature distribution. Recently, Xiong, and Yanbo [22] established a fractionalorder generalized themoelastic diffusion theory for anisotropic and linearly thermoelastic diffusive media.

In this paper, we have modified the work of Tripathi et al. [4] and Raslan [17] and studied time fractional generalized thermoelastic problem in a thick circular plate under axisymmetric temperature distribution with periodically varying heat source in the context of LordShulman theory. 
Mathematical model is prepared for Copper material and the numerical inversion of Laplace transform is performed using Gaver-Stehfast algorithm [18-20]. All the integrals involved in the problem are evaluated using Romberg integration technique [21] with variable step size. Numerical results are computed and represented graphically for temperature, displacement and stress distribution due to a periodically varying heat source using codes developed in Matlab programming environment.

\section{Formulation of the Problem}

We take the axis of symmetry as the $z$ axis and the origin of the system of co-ordinates is at the middle plane between the upper and lower faces of the plate. The problem is studied using the cylindrical polar co-ordinates $(r, \varphi, z)$. Due to the rotational symmetry about the $z$ axis, all quantities are independent of the co-ordinate $\varphi$.

Consider a thick circular plate of thickness $2 b$ occupying the space $D$ defined by

$D=\{(r, \phi, z): 0 \leq r \leq \infty,-b \leq z \leq b\}$

The problem is thus two-dimensional with all functions considered depending on the spatial variables $r$ and $Z$ as well as on the time variable $t$.

The displacement vector, thus, has the form $\vec{u}=(u, 0, w)$. The equations of motion can be written as

$\mu \nabla^{2} u-\frac{\mu}{r^{2}} u+(\lambda+\mu) \frac{\partial e}{\partial r}-\gamma \frac{\partial T}{\partial r}=\rho \frac{\partial^{2} u}{\partial t^{2}}$

$\mu \nabla^{2} w+(\lambda+\mu) \frac{\partial e}{\partial z}-\gamma \frac{\partial T}{\partial z}=\rho \frac{\partial^{2} w}{\partial t^{2}}$

The generalized equation of heat conduction has the form $[4,11]$

$$
\begin{aligned}
& k \nabla^{2} T=\left(\frac{\partial}{\partial t}+\tau_{0} \frac{\partial^{\alpha+1}}{\partial t^{\alpha+1}}\right)\left(\rho c_{E} T+\gamma T_{0} e\right) \\
& -\rho\left(1+\tau_{0} \frac{\partial^{\alpha}}{\partial t^{\alpha}}\right) Q
\end{aligned}
$$

where $T$ is the absolute temperature and $e$ is the cubical dilatation given by the relation [4]

$$
\begin{aligned}
& e=\frac{u}{r}+\frac{\partial u}{\partial r}+\frac{\partial w}{\partial z}=\frac{1}{r} \frac{\partial}{\partial r}(r u)+\frac{\partial w}{\partial z} \\
& \nabla^{2}=\frac{\partial^{2}}{\partial r^{2}}+\frac{1}{r} \frac{\partial}{\partial r}+\frac{\partial^{2}}{\partial z^{2}}
\end{aligned}
$$

The following constitutive relations supplement the above equations

$$
\begin{aligned}
& \sigma_{r r}=2 \mu \frac{\partial u}{\partial r}+\lambda e-\gamma\left(T-T_{0}\right) \\
& \sigma_{z z}=2 \mu \frac{\partial w}{\partial z}+\lambda e-\gamma\left(T-T_{0}\right) \\
& \sigma_{r z}=\mu\left(\frac{\partial u}{\partial z}+\frac{\partial w}{\partial r}\right)
\end{aligned}
$$

We shall use the following non-dimensional variables $r^{\prime}=c_{1} \eta r, z^{\prime}=c_{1} \eta z, u^{\prime}=c_{1} \eta u, w^{\prime}=c_{1} \eta w, t^{\prime}=c_{1}^{2} \eta t$

$\tau_{0}^{\prime}=c_{1}^{2 \alpha} \eta^{\alpha} \tau_{0}, \sigma^{\prime}{ }_{i j}=\frac{\sigma_{i j}}{\mu}, \theta=\frac{\gamma\left(T-T_{0}\right)}{(\lambda+2 \mu)}, Q^{\prime}=\frac{\rho \gamma Q}{k c_{1}^{2} \eta^{2}(\lambda+2 \mu)}$

where $\eta=\frac{\rho c_{E}}{k} c_{1}=\sqrt{\frac{\lambda+2 \mu}{\rho}}$ is the speed of propagation of isothermal elastic wave.

Using the above non-dimensional variables, the governing equations take the form (dropping the primes for convenience)

$\nabla^{2} u-\frac{u}{r^{2}}+\left(\beta^{2}-1\right) e-\beta^{2} \frac{\partial \theta}{\partial r}=\beta^{2} \frac{\partial^{2} u}{\partial t^{2}}$

$\nabla^{2} w+\left(\beta^{2}-1\right) \frac{\partial e}{\partial z}-\beta^{2} \frac{\partial \theta}{\partial z}=\beta^{2} \frac{\partial^{2} w}{\partial t^{2}}$

$\nabla^{2} \theta=\left(\frac{\partial}{\partial t}+\tau_{0} \frac{\partial^{\alpha+1}}{\partial t^{\alpha+1}}\right)(\theta+\varepsilon e)-\left(1+\tau_{0} \frac{\partial^{\alpha}}{\partial \mathrm{t}^{\alpha}}\right) Q$

where $\grave{o}=\frac{T_{0} \gamma^{2}}{(\lambda+2 \mu) \eta \mathrm{k}}$

The constitutive relations (6)-(8), become

$\sigma_{r r}=2 e_{r r}+\left(\beta^{2}-2\right) e-\beta^{2} \theta$

$\sigma_{z z}=2 e_{z z}+\left(\beta^{2}-2\right) e-\beta^{2} \theta$

$\sigma_{r z}=2 e_{r z}$

$\sigma_{r \varphi}=\sigma_{\varphi r}=0$

Here $\beta^{2}=\frac{(\lambda+2 \mu)}{\mu}$

Combining Eqs. (9) and (11), we obtain upon using Eq. (4),

$\nabla^{2} e-\nabla^{2} \theta=\frac{\partial^{2} e}{\partial t^{2}}$

We assume that the initial state is quiescent. The thermal and mechanical boundary conditions of the problem at $z= \pm h$ are taken as

$\sigma_{z z}(r, \pm h, t)=0$

$\sigma_{r z}(r, \pm h, t)=0$

$\theta(r, \pm h, t)=f(r, t)$

where $f(r, t)$ are known function of rand t. Eqs. (1)-(19) constitute the generalized thermoelastic formulation of the problem on a thick circular plate of infinite extent and finite thickness.

\section{Solution of the Problem}

Applying the Laplace transform defined by the relation,

$\bar{f}(r, z, s)=L[f(r, z, t)]=\int_{0}^{\infty} e^{-s t} f(r, z, t) d t$

to all the non-dimensional Eqs. (9)-(19), we get, 


$$
\begin{aligned}
& \nabla^{2} \bar{u}-\frac{\bar{u}}{r^{2}}+\left(\beta^{2}-1\right) \bar{e}-\beta^{2} \frac{\partial \bar{\theta}}{\partial r}=\beta^{2} s^{2} \bar{u} \\
& \nabla^{2} \bar{w}+\left(\beta^{2}-1\right) \frac{\partial \bar{e}}{\partial z}-\beta^{2} \frac{\partial \bar{\theta}}{\partial z}=\beta^{2} s^{2} \bar{w} \\
& \nabla^{2} \bar{\theta}=\left(\mathrm{s}+\tau_{0} s^{\alpha+1}\right)(\theta+\varepsilon \bar{e})-\left(1+\tau_{0} \mathrm{~s}^{\alpha}\right) \bar{Q} \\
& \left(\nabla^{2}-s^{2}\right) \bar{e}=\nabla^{2} \bar{\theta} \\
& \bar{\sigma}_{r r}=2 \frac{\partial \bar{u}}{\partial r}+\left(\beta^{2}-2\right) \bar{e}-\beta^{2} \bar{\theta} \\
& \bar{\sigma}_{z z}=2 \frac{\partial \bar{w}}{\partial z}+\left(\beta^{2}-2\right) \bar{e}-\beta^{2} \bar{\theta} \\
& \bar{\sigma}_{r z}=\left(\frac{\partial \bar{u}}{\partial z}+\frac{\partial \bar{w}}{\partial r}\right) \\
& \bar{\theta}_{=} \bar{f}(r, s) \\
& \bar{\sigma}_{z z}=\bar{\sigma} r z=0
\end{aligned}
$$

Eliminating $\bar{e}$ between the Eqs. (23) and (24), we get,

$$
\begin{aligned}
& \left\{\nabla^{2}-\left(s^{2}+s\left(1+\tau_{0} s^{\alpha}\right)(1+\varepsilon)\right) \nabla^{2}+s^{3}\left(1+\tau_{0} s^{\alpha}\right)\right\} \bar{\theta} \\
& =-\left(1+\tau_{0} s^{\alpha}\right)\left(\nabla^{2}-s^{2}\right) \bar{Q}
\end{aligned}
$$

After factorization the above equation can be written as,

$$
\left(\nabla^{2}-k_{1}^{2}\right)\left(\nabla^{2}-k_{2}^{2}\right) \bar{\theta}=-\left(1+\tau_{0} s^{\alpha}\right)\left(\nabla^{2}-s^{2}\right) \bar{Q}
$$

where $k_{1}{ }^{2}$ and $k_{2}{ }^{2}$ are the roots with positive real parts of the characteristic equation

$$
k^{4}-\left(s^{2}+s\left(1+\tau_{0} s^{\alpha}\right)(1+\varepsilon)\right) k^{2}+s^{3}\left(1+\tau_{0} s^{\alpha}\right)=0
$$

The solution of Eq. (31) is written in the form,

$\bar{\theta}=\bar{\theta}_{1}+\bar{\theta}_{2}+\bar{\theta}_{p}$

where $\bar{\theta}_{i}$ is a solution of the homogenous equation,

$$
\left\{D^{2}-\left(k_{\mathrm{i}}^{2}+\mathrm{q}^{2}\right)\right\} \bar{\theta}_{i}^{*}=0, i=1,2
$$

and $\bar{\theta}_{p}$ is a particular integral of Eq. (31).

In order to solve the problem, the Hankel transform of order zero with respect to $r$ is used. The Hankel transform of a function $\bar{f}(r, z, s)$ is defined by the relation,

$$
\bar{f}^{*}(\mathrm{q}, z, s)=H[\bar{f}(r, z, s)]=\int_{0}^{\infty} \bar{f}(r, z, s) r J_{0}(\mathrm{q} r) d r
$$

where $J_{0}$ is the Bessel function of the first kind of order zero and $\mathrm{q}$ is the Hankel transform parameter.

The inversion of Hankel transform is given by

$$
\begin{aligned}
& \bar{f}(r, z, s)=H^{-1}\left[\bar{f}^{*}(q, z, s)\right] \\
& =\int_{0}^{\infty} \bar{f}^{*}(q, z, s) q J_{0}(q r) d q
\end{aligned}
$$

Applying the Hankel transform to Eq. (34), we get,
$\left\{D^{2}-\left(k_{\mathrm{i}}^{2}+\mathrm{q}^{2}\right)\right\} \bar{\theta}_{i}^{*}=0, i=1,2$

where $D=\partial / \partial z$. The solution of Eq. (37) can be expressed as,

$\bar{\theta}_{i}^{*}=A_{i}(\mathrm{q}, s)\left(k_{i}^{2}-s^{2}\right) e^{-\mu_{i} z}$

where $\mu_{i}=\sqrt{\mathrm{q}^{2}+k_{i}^{2}}$

Applying Hankel transform to the Eq. (31), we get,

$\left(D^{2}-\mu_{1}^{2}\right)\left(D^{2}-\mu_{2}^{2}\right) \bar{\theta}_{p}^{*}=-\left(1+\tau_{0} \mathrm{~s}^{\alpha}\right)\left(\mathrm{D}^{2}-\mu^{2}\right) \bar{Q}^{*}$

where $\mu=\sqrt{\mathrm{q}^{2}+s^{2}}$

The periodically varying heat source $Q(r, z, t)$ in cylindrical co-ordinates is taken in the following form

$$
\begin{array}{rlrl}
Q(r, z, t) & =Q_{0} \frac{\delta(r)}{2 \pi r} \frac{\sin \pi t}{\tau}, & 0 \leq t \leq \tau \\
& =0 \quad, t>\tau
\end{array}
$$

where $Q_{0}$ is the strength of the heat source and $\delta(r)$ is the well known Dirac's delta function.

On applying Laplace transform and Hankel transforms to Eq. (40), we get,

$\bar{Q}^{*}=\frac{Q_{0} \pi \tau\left(1+e^{-s \tau}\right)}{\left(s^{2} \tau^{2}+\pi^{2}\right)}$

Using Eq. (41), we arrive at the particular integral of equation (39) as follows,

$\bar{\theta}_{p}^{*}=\frac{\left(1+\tau_{0} s^{\alpha}\right) \mu^{2}}{\mu_{1}^{2} \mu_{2}^{2}} \frac{Q_{0} \pi \tau\left(1+e^{-s \tau}\right)}{\left(s^{2} \tau^{2}+\pi^{2}\right)}$

Using Eq. (33), we obtain the complete solution for dimensionless temperature in the transformed domain as follows,

$$
\begin{aligned}
\bar{\theta}^{*}(\mathrm{q}, z, s)=A_{i}(\mathrm{q}, s)\left(k_{i}^{2}-s^{2}\right) \cosh \mu_{\mathrm{i}} \mathrm{z} \\
+\frac{\left(1+\tau_{0} s^{\alpha}\right) \mu^{2}}{\mu_{1}^{2} \mu_{2}^{2}} \frac{Q_{0} \pi \tau\left(1+e^{-s \tau}\right)}{\left(s^{2} \tau^{2}+\pi^{2}\right)}
\end{aligned}
$$

On applying the inverse Hankel transform to Eq. (43), we get,

$\bar{\theta}(r, z, s)=\int_{0}^{\infty}\left\{\begin{array}{l}\sum_{i=1}^{n} A_{i}(q, s)\left(k_{i}^{2}-s^{2}\right) \cosh \mu_{i} z \\ +\frac{\left(1+\tau_{0} s^{\alpha}\right) \mu^{2}}{\mu_{1}^{2} \mu_{2}^{2}} \frac{Q_{0} \pi \tau\left(1+e^{-s \tau}\right)}{\left(s^{2} \tau^{2}+\pi^{2}\right)}\end{array}\right\} q J_{0}(q r) d q$

Similarly, eliminating $\theta$ between Eqs. (23) and (24), we get,

$\left(\nabla^{2}-k_{1}^{2}\right)\left(\nabla^{2}-k_{2}^{2}\right) \bar{e}=-\left(1+\tau_{0} s^{\alpha}\right) \nabla^{2} \bar{Q}$

On applying Hankel transform to Eq. (45), we get, 


$$
\begin{aligned}
\left(D^{2}-\mu_{1}^{2}\right)\left(D^{2}-\mu_{2}^{2}\right) e^{-*} & \\
& =-\left(1+\tau_{0} s^{\alpha}\right)\left(D^{2}-\mu^{2}\right) \bar{Q}^{*}
\end{aligned}
$$

Complete solution of Eq. (46) can be expressed as,

$$
\begin{aligned}
\bar{e}^{*}(\mathrm{q}, z, s)=\sum_{i=1}^{2} A_{i}(\mathrm{q}, s) k_{i}^{2} \cosh \mu_{\mathrm{i}} \mathrm{z} & \\
+ & \frac{\left(1+\tau_{0} s^{\alpha}\right) \mu^{2}}{\mu_{1}^{2} \mu_{2}^{2}} \frac{Q_{0} \pi \tau\left(1+e^{-s \tau}\right)}{\left(s^{2} \tau^{2}+\pi^{2}\right)}
\end{aligned}
$$

Taking the inverse Hankel Transform of Eq. (47), we get,

$$
\bar{e}(r, z, s)=\int_{0}^{\infty}\left\{\frac{\sum_{i=1}^{2} A_{i}(q, s) k_{i}^{2} \cosh \mu_{i} z+}{\frac{\left(1+\tau_{0} s^{\alpha}\right) \mu^{2}}{\mu_{1}^{2} \mu_{2}^{2}} \frac{Q_{0} \pi \tau\left(1+e^{-s \tau}\right)}{\left(s^{2} \tau^{2}+\pi^{2}\right)}}\right\} q J_{0}(q r) d q
$$

On applying Hankel transform to Eq. (22) and then using Eqs. (43) and (47), the axial displacement component is expressed as,

$\bar{w}^{*}(\mathrm{q}, z, s)=C \sinh m z+\sum_{i=1}^{2} A_{i}(\mathrm{q}, s) \mu_{i} \sinh \mu_{\mathrm{i}} \mathrm{z}$

where $m=\sqrt{q^{2}+\beta^{2} s^{2}}$

On applying the inverse Hankel transform to Eq. (49), we get,

$\bar{w}(r, z, s)=\int_{0}^{\infty}\left[C \sinh m z-\sum_{i=1}^{2} A_{i} \mu_{i} \sinh \mu_{i} z\right] q J_{0}(q r) d q$

Let, $\bar{u}=\frac{\partial \bar{\phi}}{\partial r}, \bar{\phi}$

Taking the Laplace Transform of Eq. (4) and making use of Eq. (51), we get,

$\bar{e}=\frac{1}{r} \frac{\partial}{\partial r}\left(r \frac{\partial \bar{\phi}}{\partial r}\right)+\frac{\partial \bar{w}}{\partial z}$

On applying the Hankel transform to Eq. (52), we get,

$\bar{\phi}^{*}=\frac{1}{q^{2}}\left(\frac{\partial \bar{w}^{*}}{\partial z}-\bar{e}^{*}\right)$

On applying the inverse Hankel transform to Eq. (53), we get,

$\bar{\phi}^{*}=\int_{0}^{\infty} \frac{1}{q}\left(\frac{\partial \bar{w}^{*}}{\partial z}-\bar{e}^{-*}\right) J_{0}(q r) d q$

Substituting Eq. (54) in Eq. (51) and making use of Eqs. (48) and (49), we get,

$$
\begin{aligned}
\bar{u}=-\int_{0}^{\infty}\left[C_{m} \cosh m z+\sum A_{i} q^{2} \cosh \mu_{i} z\right. \\
\\
\left.\quad-\frac{\left(1+\tau_{0} s^{\alpha}\right) \mu^{2}}{\mu_{1}^{2} \mu_{2}^{2}} \frac{Q_{0} \pi \tau\left(1+e^{-s \tau}\right)}{s^{2} \tau^{2}+\pi^{2}}\right] J_{1}(q r) d q
\end{aligned}
$$

Substituting from Eqs. (44), (48), (49) and (54) into (26) and (27), we get,

$$
\begin{aligned}
\sigma_{z z}=\int_{0}^{\infty}\left[2 C_{m} \cosh m z\right. & +\sum A_{i}\left(q^{2}+m^{2}\right) \cosh \mu_{i} z \\
& \left.-2 \frac{\left(1+\tau_{0} s^{\alpha}\right) \mu^{2}}{\mu_{1}^{2} \mu_{2}^{2}} \frac{Q_{0} \pi \tau\left(1+e^{-s \tau}\right)}{s^{2} \tau^{2}+\pi^{2}}\right] q J_{0}(q r) d q
\end{aligned}
$$

and

$$
\begin{aligned}
\sigma_{r z}=-\int_{0}^{\infty}\left[2 \sum A_{i} q^{2} \mu_{i} \sinh \mu_{i} z\right. \\
\left.+C\left(m^{2}+q^{2}\right) \operatorname{Sinhmz}\right] J_{1}(q r) d q
\end{aligned}
$$

Also, we take,

$\bar{\theta}(r, \pm h, s)=\frac{1}{s} \theta_{0}(r)$

where, $\theta_{0}(r)=\left(\begin{array}{cc}c_{0} & 0<r<a \\ 0 & \text { otherwise }\end{array}\right.$

It implies that both the upper and lower surfaces of the thick circular plate are suddenly heated on a circle surrounding the $\mathrm{z}$-axis while the rest of the surfaces were kept at the reference temperature $\theta=0$ i.e. $T=T_{0}$.

On applying Hankel transform to equation (58), we get,

$\bar{\theta}^{*}=\frac{c_{0} a J_{1}(a q)}{s q}$

Using transformed boundary conditions given by equations (28)-(29) and making use of Eqs. (44), (56), (57) and (59), we get,

$$
\begin{aligned}
& \sum A_{i}\left(q^{2}+m^{2}\right) \cosh \mu_{i} h+2 \operatorname{Cm} \operatorname{Cosh}(m h) \\
& -\frac{2\left(1+\tau_{0} s^{\alpha}\right) \mu^{2}}{\mu_{1}^{2} \mu_{2}^{2}} \frac{Q_{0} \pi \tau\left(1+e^{-s \tau}\right)}{s^{2} \tau^{2}+\pi^{2}}=0
\end{aligned}
$$

$2 \sum A_{i} q^{2} \mu_{i} \sinh \mu_{i} h+C\left(m^{2}+q^{2}\right) \sinh (m h)=0$

$\sum_{i=1}^{2} A_{i}(q, s)\left(k_{i}^{2}-s^{2}\right) \cosh \mu_{i} z$

$+\frac{\left(1+\tau_{0} s^{\alpha}\right) \mu^{2}}{\mu_{1}^{2} \mu_{2}^{2}} \frac{Q_{0} \pi \tau\left(1+e^{-s \tau}\right)}{\left(s^{2} \tau^{2}+\pi^{2}\right)}=\frac{c_{0} a J_{1}(a q)}{s q}$

Eqs. (60)-(62) is a system of linear equations with $A_{1}(\eta, s)$, $A_{2}(\eta, s)$ and $B(\eta, s)$ as unknown parameters. On solving these equations, we get the complete solution of the problem in the transformed domain. 


\section{Inversion of Double Transforms}

The Laplace transform of a continuous function $f(t)$ is given by

$$
\bar{f}(s)=\int_{0}^{\infty} e^{-s t} f(t) d t
$$

for $t>0$ and $s=x+i y$.If the solution is given in the Laplace domain, the inversion integral is used to find the original function $f(t)$

$$
f(t)=\int_{\gamma-i \infty}^{\gamma+i \infty} e^{-s t} \bar{f}(s) d t
$$

where the contour must be taken to the right of any singularities of $\bar{f}(s)$. The direct integration of Eq. (64) is normally difficult and in many cases analytically not possible. By this method the inverse $f(t)$ of the Laplace transform $\bar{f}(s)$ is approximated by,

$$
f(t)=\frac{\ln 2}{t} \sum_{j=1}^{K} D(j, K) F\left(j \frac{\ln 2}{t}\right)
$$

with

$$
\begin{aligned}
& D(j, K) \\
& =(-1)^{j+M} \sum_{n=m}^{\min (j, M)} \frac{n^{M}(2 n) !}{(M-n) ! n !(n-1) !(j-n) !(2 n-j) !}
\end{aligned}
$$

where $K$ is an even integer, whose value depends on the word length of the computer used. $M=K / 2$ and $m$ is the integer part of the $(j+1) / 2$. The optimal value of $K$ was chosen as described in Gaver-Stehfast algorithm [23-25], for the fast convergence of results with the desired accuracy. This method is easy to implement and very accurate for functions of the type $e^{-\alpha t}$. The Romberg numerical integration technique [26] with variable step size was used to evaluate the integrals involved. All the programs were made in mathematical software Matlab.

\section{Numerical Results and Discussion}

For numerical calculations, we take

$f(r, t)=\theta_{0} H(a-r) H(t)$

where $\theta_{0}$ is a constant. On taking Hankel and Laplace transform of the above function, we get,

$\bar{f}^{*}(\alpha, s)=\frac{a \theta_{0} J_{1}(\eta a)}{\eta s}$

Copper material was chosen for purposes of numerical computations, with the physical data given as [20]

$$
\begin{aligned}
& \rho=8954 \mathrm{~kg} \cdot \mathrm{m}^{-3}, \eta=8886.73 \mathrm{~s} \cdot \mathrm{m}^{-2}, \\
& k=386 \mathrm{~J} \cdot \mathrm{K}^{-1} \cdot \mathrm{m}^{-1} \cdot \mathrm{s}^{-1}, \tau_{0}=0.025, T_{0}=293 \mathrm{~K}, \\
& \lambda=7.76 \times 10^{10} \mathrm{~N} \cdot \mathrm{m}^{-2}, \alpha_{t}=1.78 \times 10^{-5} \mathrm{~K}^{-1}, \\
& c_{E}=383.1 \mathrm{~J} \cdot \mathrm{k} \mathrm{g}^{-1} \cdot \mathrm{K}^{-1}, \mu=3.86 \times 10^{10} \mathrm{~N} \cdot \mathrm{m}^{-2}, \\
& c_{1}=4.158 \times 10^{3} \mathrm{~m} \cdot \mathrm{s}^{-1}, \varepsilon=0.0168 \mathrm{~N} \cdot \mathrm{m} \cdot \mathrm{J}^{-1}, \\
& \beta^{2}=4, a=7, b=1
\end{aligned}
$$

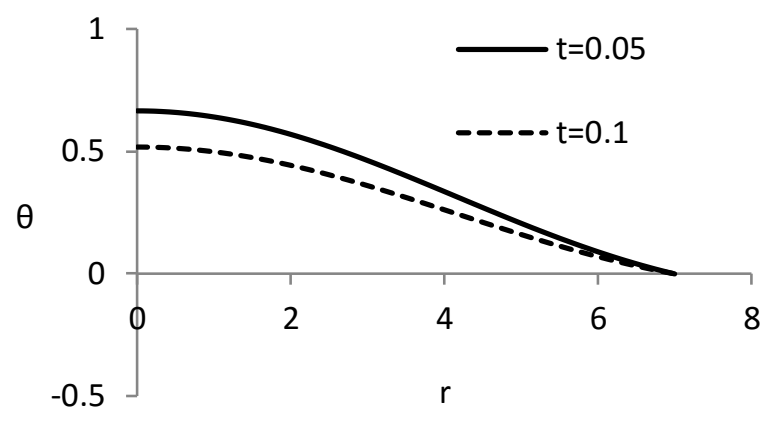

Figure 1. Temperature distribution $\theta$ in the middle plane for $\alpha=0.98$

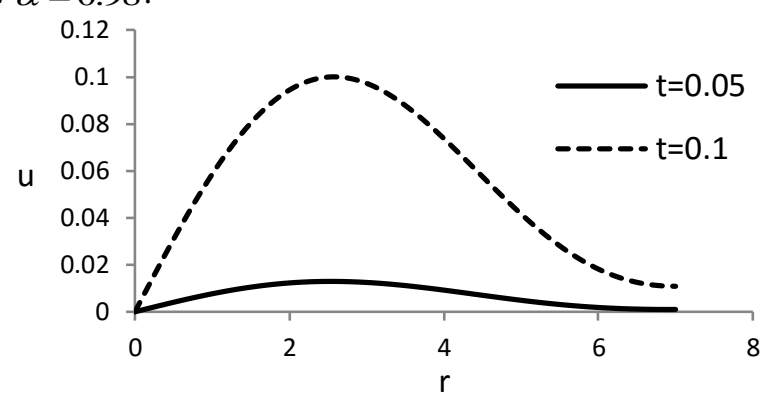

Figure 2. Radial displacement $u$ distribution in the middle plane for $\alpha=0.98$.

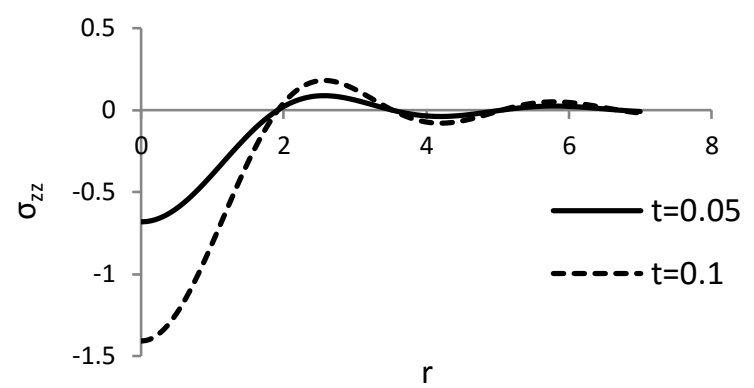

Figure 3. Axial stress component $\sigma_{z z}$ in the middle plane for $\alpha=0.98$.

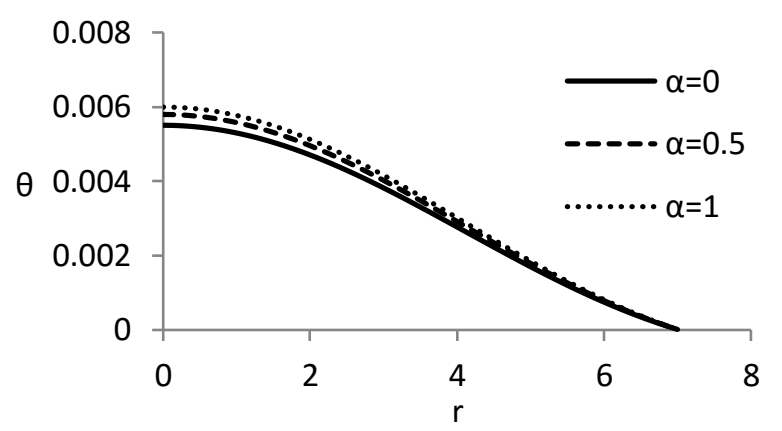

Figure 4. Temperature distribution $\theta$ in the middle plane for $t=0.05$.

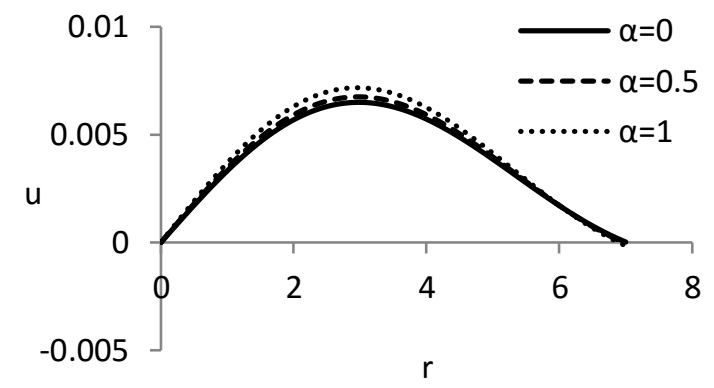

Figure 5. Radial Displacement $u$ in the middle plane for $t=0.05$ 


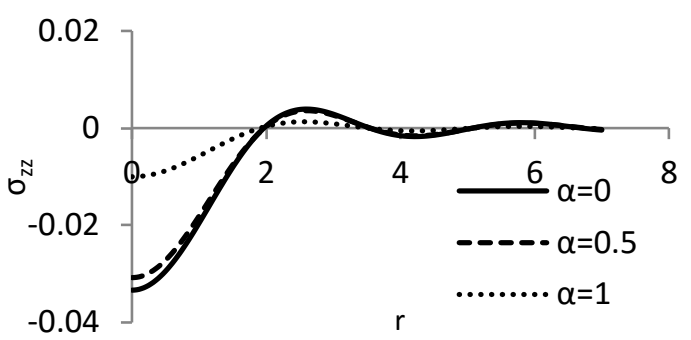

Figure 6. Axial stress component $\sigma_{z z}$ in the middle plane for $t=0.05$

Figures 1-3 exhibit the variations of $\theta$, the radial displacement component $u$ and the axial stress component $\sigma_{z z}$ considered as functions of radial distance $r$ at the middle of the plane $(z=0)$ for different time instants $t=0.05,0.1$. Since the displacement function $w$ is an odd function of $z$, its value on the middle plane is always zero and it is not represented graphically here. The value of $\alpha$ in these figures was taken equal to 0.98. In these figures, solid line represents the solutions for $t=0.05$ and dashed line represents the solutions for $t=0.1$.

Figures 4-6 depict the behavior of $\boldsymbol{\theta}, u$ and $\sigma_{z z}$ along the radial direction for the different values of fractional order parameter $\alpha$ and hence shows the variations between the generalized and fractional order thermoelasticity theories. In all these figures the solid line represents the solutions for $\alpha=0$ and dashed lines represent the solutions for $\alpha=0.5$ and 0.1 respectively.

Figure 1 shows the variation of temperature with radial distance. It is observed that the temperature decreases with radial distance and finally becomes identically zero at $r=7$. It is also observed that the values of temperature at $t=0.05$ are more as compared to its values at $t=0.1$.

Figure 2 depicts the variation of $u$ with the radial distance. It is observed that, $u$ increases with the radial distance up to $r=2.5$ and then gradually decreases till $r=7$. In the complete region, the values of $u$ for $t=0.1$ are less than its values at $t=0.05$. It is also observed that the radial displacement component $u$ becomes identically zero for $t=0.05$ at $r=7$.

Figure 3 shows the variation of axial stress $\sigma_{z z}$ with radial distance. It is observed that the axial stresses are compressive in the region $0 \leq r \leq 1.8$ and $3.6 \leq r \leq 5$. The axial stress component values are tensile in the region $1.8 \leq r \leq 3.6$ and $5 \leq r \leq 7$.

Figures 4-6 shows the variations of $\boldsymbol{\theta}, u$ and $\sigma_{z z}$ along the radial direction for different values of the fractional order parameter $\alpha$. We can clearly observe the effect of different values of $\alpha$ on the velocity of the waves and an inference can be drawn that the speed of waves is directly proportional to the values of fractional order parameter $\alpha$. Hence, an increase in the conductivity of energy in the material is directly related with the fractional order parameter. For $\alpha \cong 1$, the solutions behave like the generalized theory of thermoelasticity.

\section{Conclusion}

In this problem, we have used the time fractional order theory of thermoelasticity to solve the problem for a thick circular plate with a periodically varying heat source. A direct approach is used to solve the problem without the use of customary potential functions which helps in eliminating the well-known problems associated with the solutions using potential functions. Due to the presence of one relaxation time in the field equations the heat wave assumes finite speed of propagation. It is concluded that for different values of the fractional order parameter $\alpha$, the velocity of the wave changes. When $\alpha \cong 1$, the solutions behave like generalized thermoelasticity. The fractional order parameter seems to be directly proportional to the conductivity of the material. The system of equations in this paper may prove to be useful in studying the thermal characteristics of various bodies in real life engineering problems by considering the time fractional derivative in the field equations.

\section{Acknowledgements}

The authors are thankful to the valuable and constructive suggestions of the reviewers which have improved the manuscript.

\section{References}

[1] H. Lord, Y. Shulman, "A Generalized Dynamical theory of thermoelasticity," J. Mechanics Physics Solids, 15, 299-307, 1967.

[2] S. H. Mallik, M. Kanoria, "A Two dimensional problem for a transversely isotropic generalized thermoelastic thick plate with spatially varying heat source," European J. Mechanics A/Solids, 27, 607$621,2008$.

[3] N. M. El-Maghraby, "A two dimensional problem for a thick plate and heat sources in Generalized thermoelasticity," J. Thermal Stresses, 28, 1227-1241, 2005 .

[4] J. J. Tripathi, G. D. Kedar, K. C. Deshmukh, "Dynamic Problem of Generalized Thermoelasticity for a Semi-infinite Cylinder with Heat Sources," J. Thermoelasticity , 2, 1-8, 2014.

[5] J. J. Tripathi, G. D. Kedar, K. C. Deshmukh, "Generalized thermoelastic diffusion problem in a thick circular plate with axisymmetric heat supply," Acta Mech., 226, 2121-2134, 2015.

[6] J. J. Tripathi, G. D. Kedar, K. C. Deshmukh, “Two dimensional generalized thermoelastic diffusion in a half space under axisymmetric distributions," Acta Mech., 226, 3263-3274, 2015.

[7] Y.Z. Povstenko, "Fractional heat conduction equation and associated thermal stress," J. Thermal Stresses, 28, 83-102, 2005.

[8] Y.Z. Povstenko, "Fractional heat conduction equation and associated thermal stresses in an infinite solid with a spherical cavity," Quart. J. Mech. Appl. Math., 61, 523-547, 2008

[9] Y.Z. Povstenko, "Fractional radial diffusion in an infinite medium with cylindrical cavity," Quart. Appl. Math., 67, 113-123, 2009.

[10] Y.Z. Povstenko, "Fractional radial heat conduction in an infinite medium with a cylindrical cavity and 
associated thermal stresses," Mech. Res. Commun., doi: 10.1016/j.mechrescom. 2010.04.006.

[11]H. H. Sherief, A. El-Sayed, A. A. El-Latief, "Fractional order theory of thermoelasticity," Int. J. Solids Struct. , 47, 269-275, 2010.

[12]M. A. Ezzat, A. S. El-Karamany, "Fractional order theory of a perfect conducting thermoelastic medium," Can. J. Phys., 89, 311-318, 2011.

[13] M. A. Ezzat, A. S. El-Karamany, "Theory of Fractional order in electro-thermo-elasticity," Eur. J. Mech. A/Solids, 30, 491-500, 2011.

[14]H. H. Sherief, A. El-Sayed, A. A. El-Latief, "Fractional order theory of thermoelasticity," Int. J. Solids Struct., 47, 269-275, 2010.

[15]M. Baccher, "Deformations due to periodically varying heat sources in a reference temperature dependent thermoelastic porous material with a time free heat conduction law," IRJET, 2, 145-152, 2015.

[16]M. Islam, M. Kanoria, "Short time analysis of magnetothermoelastic wave under fractional order heat conduction law," J. Thermal Stresses, 38, 1219-1249, 2015.

[17] W. E. Raslan, "Application of Fractional order theory of thermoelasticity in a thick plate under axisymmetric temperature distribution," J. Thermal Stresses, 38, 733743, 2015.

[18] J.J. Tripathi, G.D. Kedar, K.C. Deshmukh, "Generalized thermoelastic diffusion in a thick circular plate including heat source," Alexandria Engineering J., 55, 2241-2249, 2016.
[19] N, Sarkar, "Wave propagation in an initially stressed elastic half-space solids under time-fractional order two-temperature magneto-thermoelasticity," Eur. Phys. J. Plus, 132, 154, 2017.

[20] J. J. Tripathi, G. D. Kedar, K. C. Deshmukh, "Dynamic problem offractional order thermoelasticity for a thick circular plate with finite wave speeds," J. Thermal Stresses, 39, 220-230, 2016.

[21]S. D. Warbhe, J. J. Tripathi, K. C. Deshmukh, J. Verma, "Fractional Heat Conduction in a Thin Circular Plate With Constant Temperature Distribution and Associated Thermal Stresses," J. Heat Transfer, 139, 044502, 2017.

[22]X. Chunbao, N. Yanbo, "Fractional-order generalized thermoelastic diffusion theory," Applied Mathematics Mechanics, 38, 1091-1108, 2017.

[23]D. P. Gaver, "Observing Stochastic processes and approximate transform inversion," Operations Res., 14, 444-459, 1966.

[24] H. Stehfast, "Algorithm 368, Numerical inversion of Laplace transforms," Comm. Ass'n. Comp. Mach., 13, 47-49, 1970.

[25]H. Stehfast, "Remark on algorithm 368, Numerical inversion of Laplace transforms," Comm. Ass'n. Comp., 3, 624, 1970.

[26] W. H. Press, B. P. Flannery, S. A. Teukolsky, W. A. Vetterling, Numerical Recipes, Cambridge University Press, Cambridge, the art of scientific computing, 1986. 